\title{
Single Particle Imaging with the Volta Phase Plate
}

\author{
$\underline{\text { Radostin Danev }}^{1}$, Maryam Khoshouei ${ }^{2}$ and Wolfgang Baumeister ${ }^{2}$
}

1. The University of Tokyo, Tokyo, Japan.

2. Max Planck Institute of Biochemistry, Martinsried, Germany.

The Volta phase plate (VPP) is a remarkably simple device comprising a thin $(\sim 10 \mathrm{~nm})$ amorphous carbon film positioned at the back focal plane of the objective lens (Fig. 1) [1]. The film is continuously heated at $250^{\circ} \mathrm{C}$ to prevent beam-induced contamination and enable the Volta potential effect. The strong central diffraction beam of unscattered electrons interacts with the film and modifies its surface properties leading to the creation of a Volta potential difference (the outer potential just above the film surface) between the central spot and the surrounding areas. This in turn leads to a three-dimensional electrostatic potential distribution above and below the film which when integrated along the beam path results in a phase shift difference between the central beam and the scattered beams. The effect of the central diffraction beam on the film is cumulative, producing a phase shift which increases with the electron dose (Fig. 2). The increase is not linear and slows down as the phase shift approaches $\sim \pi / 2$ [1], which coincidently is the ideal phase shift for a phase plate.

Single particle applications of the VPP have shown some very encouraging results. The improved contrast was instrumental in the near-atomic cryo-EM structure determination of peroxiredoxin-3 $(\sim 250 \mathrm{kDa})[2]$, the nucleosome $(\sim 200 \mathrm{kDa})$ [3], a calcitonin class-B GPCR $(\sim 150 \mathrm{kDa})$ [4], a human GLP-1 receptor-Gs complex $(\sim 150 \mathrm{kDa})$ [5], a human adenosine A1 receptor-Gi complex $(\sim 150 \mathrm{kDa})[6]$ and hemoglobin $(64 \mathrm{kDa})$ [7]. The VPP has the capability to extend the applicability of cryo-EM towards smaller particles and it is not unreasonable to expect that in the near future even structures under $50 \mathrm{kDa}$ could be solved to near-atomic resolutions.

Despite its advantages, the VPP has some limitations. It is difficult to use and offers less flexibility in setting up the data acquisition. It also increases the chances of unsuccessful experiments because of its sensitivity to optical alignments and electrostatic charging of the sample. Furthermore, the 10 $\mathrm{nm}$ carbon film of the phase plate scatters some of the incident electrons resulting in $\sim 15 \%$ information loss. To overcome those issues, a promising alternative is currently in development in the group of Prof. Holger Mueller at UC Berkeley - a laser-based phase plate [8]. The laser phase plate is still in a very early prototype stage, but has already demonstrated phase shift. If successfully developed, it will represent the next generation of phase plates for transmission electron microscopy.

\section{References :}

[1] R Danev et al, PNAS 111 (2014), p. 15635.

[2] M Khoshouei et al, Nature Communications 7 (2016), p. 10534.

[3] EYD Chua et al, Nucleic Acids Research 44 (2016), p. 8013.

[4] YL Liang et al, Nature 546 (2017), p. 118.

[5] YL Liang et al, Nature 555 (2018), p. 121.

[6] CJ Draper-Joyce et al, Nature 558 (2018), p. 559.

[7] M Khoshouei et al, Nature Communications 8 (2017), p. 16099.

[8] O Schwartz et al, Optics Express 25 (2017), p. 14453. 


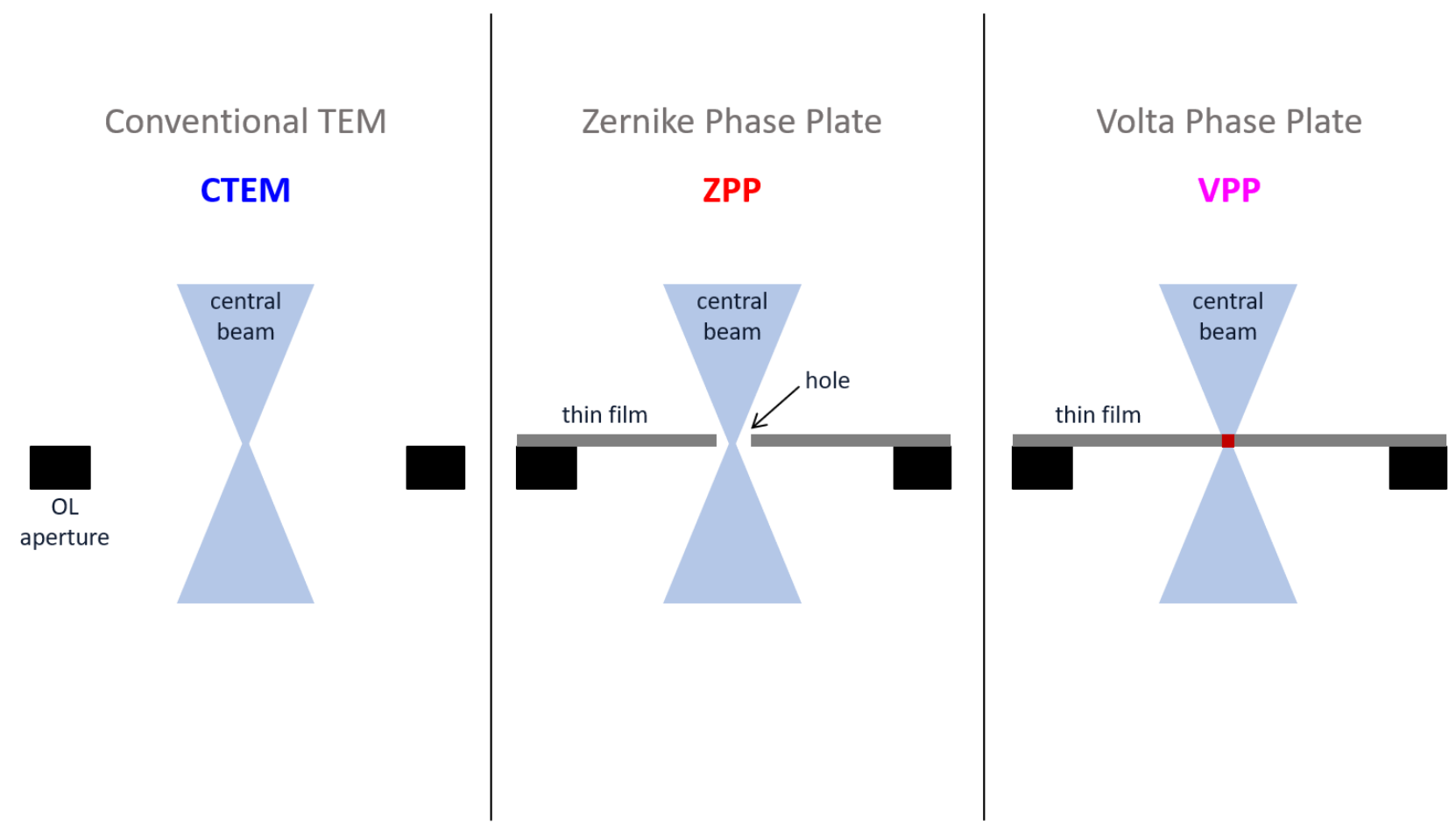

Figure 1. Illustration of the back-focal plane setup for three different imaging modes: conventional TEM (CTEM), Zernike phase plate (ZPP) and Volta phase plate (VPP).

Dose, $[\mathrm{nC}]$

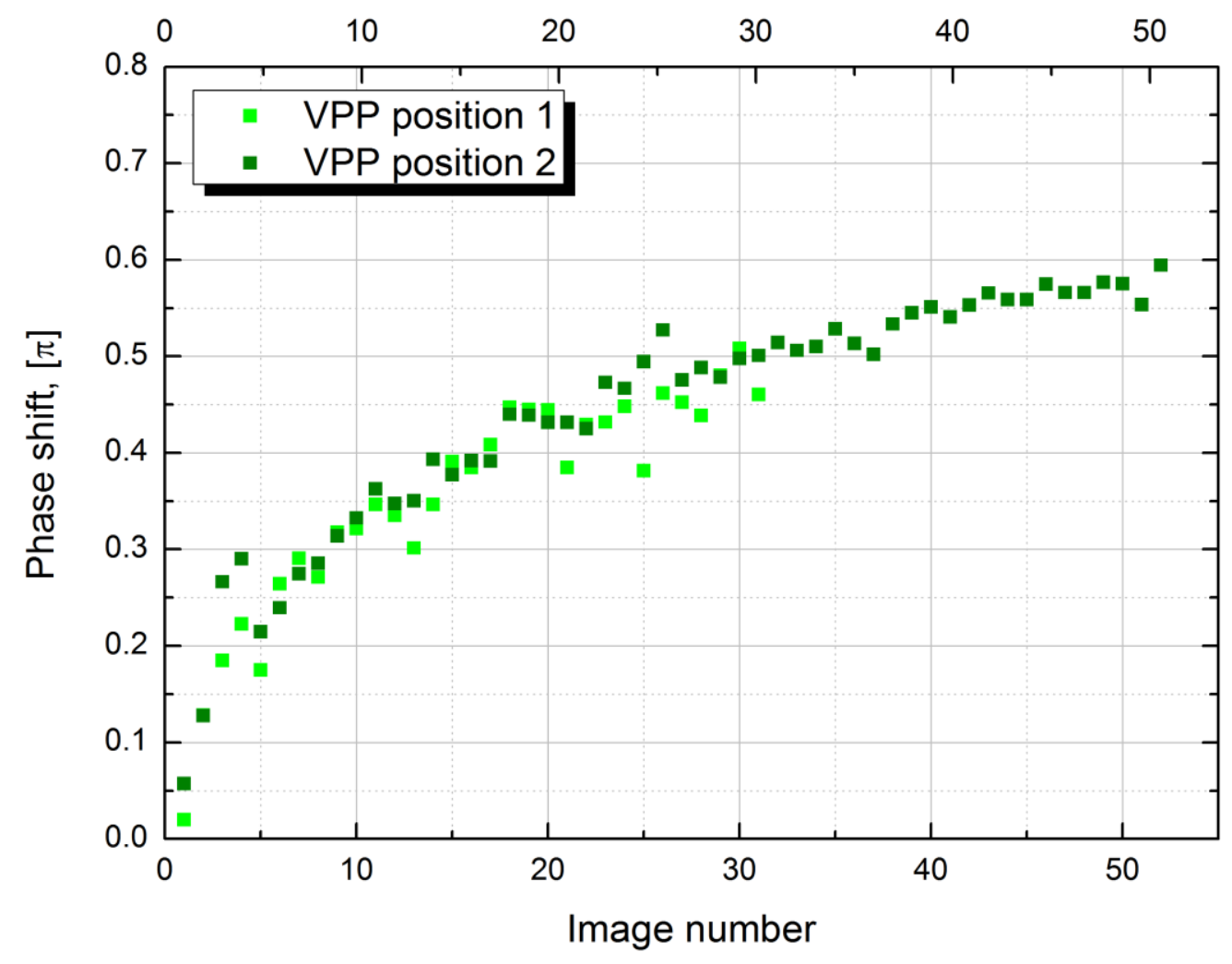

Figure 2. A plot of the phase shift development of the Volta phase plate as a function of the accumulated dose/number of images. 